\title{
Prédation des nids de deux espèces de bulbuls (Pycnonotidae) dans un écosystème de forêt tropicale humide à Korup, Sud-Ouest Cameroun
}

\author{
Jean TONLEU ${ }^{1,3^{*}}$, Kadiri Serge BOBO ${ }^{2}$, Ducelier Wamba DJOUMESSI ${ }^{3}$, Cyrille Legrand \\ Tagala TANEBANG ${ }^{2,4}$, Wilfried Gael Tiotsop LONTCHI ${ }^{2}$, Adzenkeng Célestine \\ TANYIMAJOB $^{5}$, Larissa Kehegam MFENDEM ${ }^{1}$, Rodrigue Steve Hermann ASSOUA ${ }^{6}$, \\ Urbain Talongong NZITOUO ${ }^{7}$ et MPOAME MBIDA ${ }^{3}$
}

\footnotetext{
${ }^{I}$ Ecole Nationale Supérieure Polytechnique de Maroua, Université de Maroua, BP 46 Maroua, Cameroun. ${ }^{2}$ Département de Foresterie, Faculté d'Agronomie et des Sciences Agricoles, Université de Dschang, BP 222 Dschang, Cameroun.

${ }^{3}$ Département de Biologie Animale, Faculté des Sciences, Université de Dschang, BP 67 Dschang, Cameroun.

${ }^{4}$ Parc National de Korup, Délégation Régionale du Ministère des Forêts et de la Faune du Sud-Ouest du, BP 101 Mundemba, Cameroun.

${ }^{5}$ Délégation Départementale du Ministère des Forêts et de la Faune du Moungo, BP 178 Nkongsamba, Cameroun.

${ }^{6}$ Ingénieur Statisticien en service à la Délégation Régionale de l'Extrême Nord du Ministère de la Santé Publique, BP 620 Maroua, Cameroun.

${ }^{7}$ Parc National de Campo Ma'an, Délégation Régionale du Ministère des Forêts et de la Faune du Sud, BP 19 Campo, Cameroun.

*Auteur correspondant, E-mail: tonleutommy6@yahoo.fr; Téléphone: +237655003310
}

\section{REMERCIEMENTS}

Les auteurs remercient le Projet Volkswagen "Managing Forest Wildlife for Human Livelihoods in the Korup-Oban Hills region, West- Central Africa » pour le soutien financier et IDEA WILD pour le don d'équipements.

\section{RESUME}

Avec la déforestation croissante dans les zones tropicales, les bulbuls comptent parmi les espèces d'oiseaux les plus vulnérables en raison de la perte des sites de nidification dans les habitats fortement perturbés. La présente étude vise à évaluer le niveau de prédation des nids de deux espèces de bulbuls Eurillas latirostris et Eurillas virens suivant les types d'habitats (forêts naturelles et habitats perturbés) et les phases de nidification. Dix-neuf nids actifs des bulbuls verdâtres (Eurillas virens) et 13 nids de bulbuls à moustaches jaunes (Eurillas latirostris) ont été localisés et les incidences de prédation ainsi que les paramètres liés à la reproduction de ces oiseaux ont été enregistrés pendant leur période de nidification à la périphérie Nord-Est du Parc National de Korup. L'étude révèle que la prédation est la principale cause d'échec de nidification chez ces espèces et affecte plus de $50 \%$ de nids. Les types d'habitat n'ont pas d'influences significatives sur les différents taux de prédation. Parmi les variables de la végétation, la surface terrière influence positivement le 
taux de prédation quotidien des nids de Eurillas latirostris pendant que la hauteur maximale du couvert végétal au-dessus du nid influence négativement le taux de prédation quotidien des nids de Eurillas virens. Globalement, les deux espèces ont été relativement plus vulnérables à la prédation pendant la phase d'incubation. Ces résultats soulignent la nécessité d'accorder une attention particulière aux bulbuls, ainsi qu'à d'autres groupes particulièrement sensibles à la destruction des forêts tropicales humides, dans le cadre de l'élaboration des stratégies de conservation qui promeuvent la réduction des impacts d'exploitation forestière. (C) 2018 International Formulae Group. All rights reserved.

Mots clés: Cameroun, Eurillas latirostris, Eurillas virens, Forêt naturelle, Habitats perturbés, Prédation des nids.

\title{
Predation of nests of two species of bulbuls (Pycnonotidae) in a rainforest ecosystem of Korup, South-West Cameroon
}

\begin{abstract}
With the growing deforestation in the tropical areas, bulbuls are among the most vulnerable bird species due to nesting sites lost in the heavily disturbed habitats. The current study aimed at evaluating the predation rates of two species of bulbuls, Yellow-whiskered greenbul Eurillas latirostris and Little greenbul Eurillas virens nests according to the types of habitat (natural forests and modified habitats) and the nesting phases. Nineteen nests of the Little greenbul and 13 nests of Yellow-whiskered greenbul were localized and predation incidences as well as some reproductive parameters of these birds were recorded during their nesting period in the North-Eastern border of Korup National Park. Results show that the predation is the principal cause of nest failure of these species with and affects more than $50 \%$ of nests. The types of habitat do not have significant influences on different predation rates. The basal area had a positive and significant influence on the daily predation rate of Yellow-whiskered greenbul nests while the maximum height of the vegetation cover above the nest had a negative and significant influence on the daily predation rate of the Little greenbul nests. Globally, both species are relatively and highly vulnerable to predation during the incubation phase. These results highlight the need to pay attention to bulbuls, as well as other particularly sensitive groups in tropical rainforests, when elaborating conservation strategies that promote the reduction of logging impacts.
\end{abstract}

(C) 2018 International Formulae Group. All rights reserved.

Keywords: Cameroon, Eurillas latirostris, Eurillas virens, Modified habitats, Natural forest, Nest Predation.

\section{INTRODUCTION}

La prédation des nids est la principale cause des échecs de nidification chez de nombreuses espèces d'oiseaux (Brawn et al., 2011; Fu et al., 2016; Kougoum et al., 2017, Tonleu et al., 2018) et particulièrement chez les passereaux dans plusieurs zones tropicales (Githiru et al., 2005; Newmark et Stanley, 2011). En plus, le système d'utilisation des terres dans un paysage forestier peut aussi influencer le succès de nidification indépendamment de l'étendue de la perturbation (Rodewald et Yahner, 2001). En effet, la fragmentation et la conversion des paysages forestiers peut augmenter l'intensité de prédation des nids en favorisant l'abondance et la richesse des généralistes et de prédateurs induits (Vergara et Simonetti, 2003). Ainsi, certaines études ont montré que le taux de prédation s'accroit suivant le gradient de destruction croissant des habitats (Vergara et Simonetti, 2003). Cependant Bobo (2007) a trouvé que le gradient de destruction croissante des habitats n'avait pas d'effet 
significatif sur le taux de prédation des nids artificiels.

La perte d'œufs causée par les prédateurs est particulièrement prononcée chez les oiseaux nichant dans le sous-bois comme les bulbuls parce que leurs œufs sont accessibles à un large éventail de prédateurs (Ekanayake et al., 2015; Bellamy et al., 2017). L'évaluation de l'influence des types d'habitat et de l'influence du gradient croissant de destruction des forêts sur le taux de prédation des nids a toujours été un travail très compliqué à cause des difficultés à découvrir et à suivre les nids dans les forêts tropicales (Tewksbury et al., 2006; Newmark et Stanley, 2011). Les études incluant la prédation des nids des oiseaux qui nidifient dans le sousbois ont plus été faites dans la zone américaine (Robinson et al., 2000; Mezquida et Marone, 2001; Ryder et al., 2008; Brawn et al., 2011). Rares sont celles qui concernent l'Afrique subsaharienne (Bobo et Waltert, 2011; Newmark et Stanley, 2011; Tonleu et al., 2018) et la zone Guinéo-congolienne (Bobo, 2007). Très peu d'études concernant la prédation des oiseaux du sous-bois ont intégré l'influence des types d'habitat et seulement les nids artificiels étaient utilisés (Weidinger, 2000; Vergara et Simonetti, 2003).

La présente étude vise à évaluer le taux de prédation des nids des espèces Eurillas latirostris et Eurillas virens suivant les types d'habitat (forêts naturelles et habitats perturbés) et suivant les phases de nidification. Dans ces analyses, (1) les taux de prédation quotidien (TPQ) des nids de ces oiseaux entre les types d'habitat ont été comparés; (2) l'influence des variables de la végétation sur les TPQ de ces espèces a été évaluée; et (3) les variables de l'habitat qui expliquent mieux la variation du TPQ de ces espèces dans cet écosystème forestier ont été déterminées.

\section{MATERIEL ET METHODES}

\section{Description de la zone d'étude}

La zone d'étude (se trouvant à proximité des villages Mgbegati, Abat et Basu) est située entre les coordonnées $5^{\circ} 21^{\prime} 18^{\prime \prime}-5^{\circ} 25^{\prime} 38^{\prime \prime} \mathrm{N}$ et $9^{\circ} 06^{\prime} 29^{\prime \prime}-9^{\circ} 15^{\prime} 07^{\prime \prime}$ E. Elle est localisée à la périphérie Nord-Est du Parc National de Korup (PNK) dans la Région du Sud-Ouest du Cameroun et est une entité légale du PNK (Figure 1). C'est la seule grande forêt d'Afrique centrale occidentale qui s'étend à l'origine du delta du Niger vers l'Est jusqu'au Cameroun et vers le Sud à travers la Guinée équatoriale et le Gabon. Situé au centre du refuge de la forêt Guinéocongolaise, Korup contient quatre associations forestières différentes (forêt Biafra de l'Atlantique, forêt marécageuse, forêt de Piémont et forêt Submontagnarde) (Thomas, 1995). La zone d'étude se trouve dans la forêt de Piedmont. La culture itinérante est pratiquée dans les zones agricoles associées aux différents types de forêt. Des cultures vivrières et commerciales $\mathrm{y}$ sont produites. Cette zone englobe les types d'habitat suivants: les forêts naturelles qui sont constituées des forêts primaires (FOP) avec très peu ou pas d'activités anthropiques et des forêts secondaires (FOS) où les impacts anthropiques sont présents mais plus que dans les FOP; les habitats perturbés qui sont constitués des forêts perturbées (DFO) où l'exploitation forestière y a été récemment réalisée (moins de cinq ans), des plantations de cacao / café (PCC) avec peu d'arbres naturels restants et les champs de culture annuelle (CCA) où la terre a été utilisée pour la production des cultures vivrières (manioc, igname, maïs, arachide, etc.), avec presque aucun arbre naturel laissé. Chacun des habitats suscités (FOP, FOS, DFO, PCC et CCA) a constitué une strate (Figure 1). L'avifaune est typique de la forêt pluviale de basse altitude, avec plus de 184 espèces restreintes à ce biome (Fishpool et al., 2001) et 420 espèces enregistrées (Rodewald et al., 1994). Ces 
groupes sont particulièrement diversifiés mais constitués essentiellement des passereaux (Muscicapidae, Nectariniidae, Ploceidae et Sylviidae).

\section{Collecte des données}

$\mathrm{Au}$ vu de la répartition inégale des habitats dans la zone, dans chaque type d'habitat (strate), six parcelles de $200 \mathrm{~m}$ x 200 $\mathrm{m}$, pour un total de 30 parcelles ont été installées au jugé (Scherrer, 1984) avec une distance minimale de $500 \mathrm{~m}$ entre celles-ci (Bobo, 2007) (Figure 1). Ces parcelles ont servi à la caractérisation des paramètres de la végétation et à la recherche des nids naturels d'oiseaux.

Pendant la période de reproduction de début avril à fin août 2013 chaque jour de la semaine, dépendant des conditions climatiques, tous les sites d'étude (parcelles) ont été explorés minutieusement et les nids des espèces de bulbuls ont été localisés sur des supports (plantes du sous-bois comme les arbustes, les herbacées et les lianes) construits entre 0 et $2 \mathrm{~m}$ de hauteur. Cette localisation a été faite directement ou indirectement grâce à l'observation du comportement des oiseaux adultes (Brawn et al., 2011). Les guides d'identification des oiseaux (Borrow et Demey, 2008) ont donc été utilisés à la suite de ces observations pour confirmer l'identification des espèces.

Les nids ont été suivis tous les trois jours (Buler et Hamilton, 2000). À chaque visite, les nids ont été scrutés dans le but de découvrir les signes de prédation comme: les individus (ou œufs) manquants, les morts, les oiselets partiellement attaqués par les prédateurs, les œufs cassés et les nids détruits (Dion et al., 2000). Il avait été supposé que l'oiselet aurait quitté le nid si les signes de prédation avaient été absents et si les oisillons avaient été prêts à quitter le nid lors de la précédente visite (Dion et al., 2000). Les nids ont été contrôlés à distance et le plus rapidement possible pour minimiser les perturbations et les risques de prédation dues à l'activité humaine (Martinez et al., 2004). Le nid a été qualifié de «actif» si une augmentation du nombre d'œufs, l'incubation et ou l'encadrement des nourrissons par les parents ont été observés; de «non actif » si à la suite d'une phase confirmée, rien n'était observé après plusieurs visites; de «réussi » lorsque l'envol des jeunes a été observé près du nid (Li et Martin, 1991).

Dans l'optique de vérifier l'hypothèse selon laquelle les variables de la végétation influenceraient le taux de prédation quotidien, les variables de l'habitat autour du nid ont été quantifiés suivant deux échelles. L'une s'est portée sur la plante qui supporte le nid, l'emplacement du nid sur la plante et l'environnement immédiat du lieu de nidification (microhabitat), et l'autre sur l'ensemble de la végétation autour du nid (macrohabitat) (Mezquida, 2004).

Au niveau du microhabitat, les données ont été collectées suivant les adaptations des méthodes de Dion et al. (2000) et de Wray et Whitmore (1979). Dans un premier temps, à la fin de la période de nidification, quatre bambous de $1 \mathrm{~m}$ chacun, servant de bâtons d'échantillonnage, ont été positionnés horizontalement au sol autour du nid, de façon à former une parcelle carrée avec le nid au centre. Par la suite, quatre bambous de $2 \mathrm{~m}$ de hauteur ont été fixés verticalement aux quatre angles du carré précédemment formé. Ensuite, six indicateurs de la végétation du site de nidification ont été enregistrés: la hauteur maximale de la végétation au-dessus du nid estimée en observant le dernier contact de la plante avec un bambou de $1 \mathrm{~m}$ placé verticalement sur le nid; la densité horizontale des arbres dans le carré et la visibilité totale du nid (au sol et sur l'arbuste) en utilisant la méthode de Remeš (2005); le pourcentage de la végétation couvrant le nid qui a été estimé visuellement avec 5\% comme mesure minimale; le type de végétation pour les nids au sol; la hauteur du nid sur la plante 
qui a été aussi mesurée pour les nids posés sur les arbustes; et le type de végétation supportant le nid a été noté.

A l'échelle du macrohabitat, les variables de la végétation ont été mesurés suivant l'adaptation de la méthode de Bobo et al. (2006). Contrairement à cette méthode, 30 stations d'échantillonnage de $200 \mathrm{~m}$ x $200 \mathrm{~m}$ correspondant à nos parcelles précédemment délimitées ont été utilisées au lieu de $100 \mathrm{~m} \mathrm{x}$ $100 \mathrm{~m}$. Chaque parcelle a été divisée en sousparcelle de $100 \mathrm{~m}^{2}(10 \mathrm{~m} \times 10 \mathrm{~m})$ soit 400 sous-parcelles par parcelle. Les sous-parcelles représentaient les unités potentielles d'échantillonnage. L'écartement entre les lignes était de $50 \mathrm{~m}$, et sur une même ligne les unités d'échantillonnages étaient équidistantes de $30 \mathrm{~m}$. Chaque ligne était décalée respectivement de 5 et $10 \mathrm{~m}$ à gauche et à droite. La circonférence à hauteur de poitrine a été mesuré à l'aide d'un ruban métrique qui a été placé autour des arbres (ayant un diamètre supérieur ou égal à $10 \mathrm{~cm}$ ) et à 1,30 $\mathrm{m}$ au-dessus du sol. La circonférence obtenue sur la graduation a été divisée par la valeur $\boldsymbol{\pi}$ $(3,14)$ pour avoir le diamètre qui a permis de calculer la surface terrière. Lorsque l'arbre avait des contreforts, le ruban métrique était placé à $30 \mathrm{~cm}$ au-dessus des contreforts (Newton, 2007).

Un échantillonnage systématique dans chaque parcelle avec un taux de sondage de 5\% (soit 20 sous-parcelles par unité d'échantillonnage) a été réalisé. Ainsi, 120 unités d'échantillonnage ont été inventoriées par habitat et pour un total de 600 unités d'échantillonnage dans le massif tout entier. Pour une bonne représentativité, les unités d'échantillonnage ont été disposées dans le quadrat en quatre lignes de cinq sous quadrats chacune.

La densité des arbres a été obtenue sur le terrain grâce aux mesures faites dans les sousparcelles de $10 \mathrm{~m}$ x $10 \mathrm{~m}$. Ainsi, avec l'aide d'un prospecteur, tous les arbres de diamètre supérieur ou égal à $10 \mathrm{~cm}$ contenus dans les sous-parcelles de chaque habitat ont été identifiés et comptés. À la fin, le nombre d'individus par hectare a été déduit connaissant déjà le nombre d'individus inventorié dans la sous-parcelle comme dans Bobo et al. (2006). Pour les herbacées et les ligneux de hauteur inférieure à $1,30 \mathrm{~m}$, une surface d'un $\mathrm{m}^{2}$ au centre de chaque sousparcelle a été délimitée et à l'intérieur de cette dernière, les effectifs des plantes herbacées et les petits ligneux ont été enregistrés selon la méthode de Bobo et al. (2006).

La couverture de la canopée a été estimée grâce à la méthode de Clark et Clark (1992). En effet, c'est une méthode de simple estimation visuelle de la fermeture de la canopée selon une échelle standard à sept classes (Classe 1, Classe 1,5, Classe 2, Classe 2,5, Classe 3, Classe 4, Classe 5) suivant le degré d'illumination de la couronne ou canopée (dépendant des angles de pénétration des rayons du soleil à travers la canopée).

\section{Analyse des données}

Pour évaluer l'effet des types d'habitat sur le nombre de nids ayant subi la prédation, le Test exact de Fischer a été utilisé comme dans Agresti (2002). Ce test est utilisé pour comparer les échantillons de faible taille dans un tableau de contingence $(2 \times 2)$. Le test de Chi deux d'égalité de proportion (Yandell, 1997) a été aussi utilisé pour détecter les différences de proportions des nids ayant subi la prédation d'une phase de nidification à l'autre.

Le taux de prédation quotidien (TPQ) des nids a été évalué pour chaque type d'habitat suivant le modèle logistique d'exposition (Shaffer, 2004). Le taux de prédation des nids a été défini comme le TPQ puissance la période d'exposition (environ 36 jours) constituée des périodes d'incubation et d'élevage des jeunes (Mayfield, 1975). Dans le logiciel R, il est implémenté suivant le lien complémentaire log-log (fonction « cloglog») 
grâce au package MASS (Venables and Ripley, 2002).

Le modèle linéaire généralisé à effet mixte a été utilisé pour évaluer l'influence des paramètres de l'habitat sur le TPQ. Le test de normalité (Conover, 1972) a été fait sur tous les paramètres quantitatifs. Lorsque ce dernier n'avait pas été significatif pour une variable quelconque, il était centré et réduit pour résoudre le problème de biais ou de surdispersion (la distribution des données ne suivant pas la loi normale) dû aux échelles de ces variables. Ce modèle a été estimé en utilisant le package lme4 (Bates et al., 2014), avec le TPQ comme la variable réponse, et un seul niveau de classification (Habitats) incorporée de manière hiérarchique comme effet aléatoire.

L'Akaike's Information Criterion (AIC) a été retenu pour déterminer le meilleur modèle d'ajustement en utilisant la fonction «aitab» dans le package AICcmodavg (Mazerolle, 2016). Le meilleur modèle a été défini comme celui ayant un poids AIC plus grand que ceux des modèles alternatifs (Symonds et Moussalli, 2011) ou bien AICc plus petit que les autres tel que décrit par Burnham et Anderson (2003). Le test de somme des rangs de Kruskal-Wallis (Hollander et al., 2013) a été utilisé pour tester l'influence des types d'habitat sur le TPQ.



Figure 1: Présentation de la zone d'étude. 


\section{RESULTATS}

\section{Proportions de nids victimes de prédation suivant les types d'habitat}

Quatre-vingt-huit observations ont été faites sur 19 nids de l'espèce Eurillas virens et 51 observations sur 13 nids de l'espèce Eurillas latirostris dans les habitats perturbés et les habitats naturels. Ainsi, 15 nids de l'espèce Eurillas virens ont été suivis dans les habitats naturels contre quatre nids dans les habitats perturbés. Cependant, six nids de l'espèce Eurillas latirostris ont été suivis dans les habitats perturbés contre sept nids dans les habitats naturels.

Chez l'espèce Eurillas virens, 53\% des nids ont subi la prédation dont $90 \%$ de prédation des œufs et $10 \%$ de prédation des oisillons. Outre la prédation, la désertion des nids par les parents lors de l'élevage des oisillons (9\% d'échec) est aussi une cause d'échec de nidification chez cette espèce. En revanche, chez l'espèce Eurillas latirostris $54 \%$ des nids ont subi la prédation parmi lesquels $83 \%$ de prédation des œufs et $17 \%$ de prédation des oisillons. Aussi, chez cette dernière, la désertion des nids représente $12,5 \%$ d'échec et les activités humaines $12,5 \%$ d'échec.

Chez l'espèce Eurillas virens, la proportion des nids ayant subi la prédation dans les habitats naturels (60\%) est supérieure mais pas significativement différente de la proportion de ceux des habitats perturbés $(25 \%)$ (Test exact de Fisher, $p=0,3$ ) (Tableau 1). Aussi, la proportion des nids ayant subi la prédation dans les habitats naturels $(57,14 \%)$ est supérieure mais pas significativement différente de la proportion des nids à l'intérieur des habitats perturbés (33,33\%)) (Test exact de Fisher, $p=0,6$ ) chez Eurillas latirostris (Tableau 1).

\section{Influences du type d'habitat et des variables de la végétation sur le taux de prédation quotidien}

Les types d'habitat n'ont pas une influence significative sur les taux de prédation quotidiens (TPQ) des nids des espèces Eurillas latirostris et Eurillas virens (Tableau 2).

La surface terrière et la hauteur maximale de la végétation au-dessus du nid sont les variables de la végétation qui expliquent la variation du TPQ chez Eurillas latirostris $(\triangle \mathrm{AICc} \leq 2)$ (Tableau 3). $\mathrm{La}$ visibilité totale du nid et la hauteur maximale de la végétation au-dessus du nid $(\triangle \mathrm{AICc} \leq 2)$ sont les variables de la végétation qui expliquent le mieux la variation du TPQ chez Eurillas virens.

Le modèle linéaire généralisé à effet mixte a montré que seule la surface terrière influence significativement le TPQ des nids de Eurillas latirostris (Tableau 4). Ainsi, l'accroissement de la valeur de la surface terrière entraîne une forte augmentation de la côte de probabilité de prédation $(0,134)$ des nids de cette espèce dans l'intervalle 0,85 à 1 (Figure 2 (A)). Le TPQ des nids de Eurillas virens est significativement influencé par la hauteur maximale de la végétation au-dessus du nid. De ce fait, plus la valeur de la hauteur maximale de la végétation au-dessus du nid augmente, la côte de probabilité de prédation des nids de cette espèce diminue $(-4,827 \mathrm{e}-02)$ dans l'intervalle 0 à 0,35 (voir Figure 2 (B)).

\section{Prédation des nids suivant les phases de nidification}

Les proportions de prédation ont varié considérablement le long du processus de reproduction chez toutes les deux espèces. En effet, chez les deux espèces, ces proportions étaient très élevées durant la phase d'incubation des aufs et basses durant les phases de ponte et d'élevage des oisillons (Figure 3). Ainsi, Chez Eurillas virens, les proportions de prédation des nids ont varié significativement d'une phase de nidification à une autre $\left(\chi^{2}=154,67 ; d f=2 ; p<0,001\right)$; le même phénomène a été observé chez Eurillas latirostris $\left(\chi^{2}=133,34 ; d f=2 ; p<0,001\right)$. 
Tableau 1: Nombre de nids victimes de prédation suivant les types d'habitat.

\begin{tabular}{|c|c|c|c|c|c|c|c|c|c|c|c|c|c|}
\hline \multirow{4}{*}{ Espèces } & \multicolumn{12}{|c|}{ Nombre de nids suivis } & \multirow{4}{*}{$\begin{array}{l}\text { Valeur } \\
\text { de } P\end{array}$} \\
\hline & \multicolumn{4}{|c|}{ Habitats naturels } & \multicolumn{4}{|c|}{ Habitats perturbés } & \multicolumn{4}{|c|}{ Total } & \\
\hline & \multicolumn{2}{|c|}{ Prédation } & \multicolumn{2}{|c|}{$\begin{array}{c}\text { Pas de } \\
\text { Prédation }\end{array}$} & \multicolumn{2}{|c|}{$\begin{array}{c}\text { Prédatio } \\
\mathbf{n}\end{array}$} & \multicolumn{2}{|c|}{$\begin{array}{c}\text { Pas de } \\
\text { Prédation }\end{array}$} & \multicolumn{2}{|c|}{ Prédation } & \multicolumn{2}{|c|}{$\begin{array}{c}\text { Pas de } \\
\text { Prédation }\end{array}$} & \\
\hline & $\begin{array}{l}\mathbf{N} \\
\mathbf{b}\end{array}$ & $\%$ & $\mathbf{N b}$ & $\%$ & $\mathbf{N b}$ & $\%$ & $\mathbf{N b}$ & $\%$ & $\begin{array}{l}\mathbf{N} \\
\mathbf{b}\end{array}$ & $\%$ & $\mathbf{N b}$ & $\%$ & \\
\hline $\begin{array}{l}\text { Eurillas } \\
\text { latirostris }\end{array}$ & 4 & 57,14 & 3 & $\begin{array}{c}42,8 \\
6\end{array}$ & 2 & $\begin{array}{c}33,3 \\
3\end{array}$ & 4 & 66,66 & 6 & 46 & 7 & 54 & $\mathbf{0 , 3}$ \\
\hline Eurillas virens & 9 & 60 & 6 & 30 & 1 & 25 & 3 & 75 & 10 & 53 & 9 & 47 & 0,6 \\
\hline
\end{tabular}

Notes: Nb: Nombre; \%: Pourcentage

Tableau 2: Taux de prédation quotidien et variables de la végétation suivant les types d'habitat. Les valeurs sont les moyennes \pm Ecart Type.

\begin{tabular}{cccccc}
\hline \multirow{2}{*}{ Espèces } & \multicolumn{2}{c}{ Moyenne du Taux de Prédation Quotidien } & \multicolumn{3}{c}{$\begin{array}{c}\text { Résultats du test de } \\
\text { Kruskal-Wallis }\end{array}$} \\
\cline { 2 - 6 } & Forêts naturelles & Habitats perturbés & $\boldsymbol{\chi}^{2}$ & $\boldsymbol{d f}$ & $\boldsymbol{P}$-Value \\
\hline Eurillas latirostris & $0,313 \pm 0,473$ & $0,02 \pm 0,029$ & 0,06 & 1 & 0,81 \\
Eurillas virens & $0,237 \pm 0,433$ & $0,078 \pm 0,134$ & 1.45 & 1 & 0,229 \\
\hline \multicolumn{2}{c}{ Variables de la végétation } & & & \\
\hline Variables & Moyenne des variables de la végétation & - & - & - \\
\hline Densité des plantes & $0,045 \pm 0,002$ & $0,02 \pm 0,003$ & - & - & - \\
(individus $\left./ \mathrm{m}^{2}\right)$ & & & & & - \\
Surface terrière $\left(\mathrm{m}^{2} / \mathrm{ha}\right)$ & $61,86 \pm 18,28$ & $23,387 \pm 10,01$ & - & - & - \\
\hline
\end{tabular}

Tableau 3: Meilleurs modèles d'ajustement expliquant la variation de taux de prédation quotidien chez les espèces Eurillas latirostris et Eurillas virens.

\begin{tabular}{ccccc}
\hline Modèles & K & AICc & DAICc & $\boldsymbol{w}_{\boldsymbol{i}}$ \\
\hline \multicolumn{5}{c}{ Eurillas latirostris } \\
\hline $\mathrm{M}_{\mathrm{H}+\mathrm{a}+\mathrm{b}}$ & 2 & 46,58 & 0,00 & 0,49 \\
$\mathrm{M}_{\mathrm{H}+\mathrm{a}+\mathrm{b}+\mathrm{c}}$ & 3 & 47,56 & 0,98 & 0,30 \\
$\mathrm{M}_{\mathrm{H}+\mathrm{a}+\mathrm{b}+\mathrm{c}+\mathrm{d}}$ & 8 & 49,67 & 3,09 & 0,10 \\
$\mathrm{M}_{\mathrm{H}+\mathrm{a}}$ & 1 & 49,74 & 3,16 & 0,10 \\
\hline \multicolumn{5}{c}{ Eurillas virens } \\
\hline $\mathrm{M}_{\mathrm{H}+\mathrm{d}+\mathrm{b}}$ & 8 & 75,62 & 0,00 & 0,45 \\
$\mathrm{M}_{\mathrm{H}+\mathrm{d}+\mathrm{b}+\mathrm{e}}$ & 9 & 76,77 & 1,15 & 0,25 \\
$\mathrm{M}_{\mathrm{H}+\mathrm{d}+\mathrm{b}+\mathrm{c}+\mathrm{d}}$ & 10 & 78,05 & 2,43 & 0,13 \\
$\mathrm{M}_{\mathrm{H}+\mathrm{d}}$ & 7 & 78,21 & 2,59 & 0,12 \\
$\mathrm{M}_{\mathrm{H}+\mathrm{d}+\mathrm{b}+\mathrm{e}+\mathrm{f}+\mathrm{g}}$ & 11 & 80,35 & 4,73 & 0,04
\end{tabular}

Notes: K est le nombre de paramètres du model, Loge(L) est la valeur maximisée de la fonction log-vraisemblance, AICc est le Critère d'Information d'Akaike ajusté pour un biais de petit échantillon, $\triangle \mathrm{AICc}$ est la valeur mise à l'échelle de AICc et $\boldsymbol{w}_{i}$ est le poids Akaike. Plusieurs modèles candidats ont combiné les effets du type d'habitats $(\mathrm{H})$, de la surface terrière (a), la hauteur maximale de la végétation au-dessus du nid (b), la densité des plantes du sous-bois (c), la visibilité totale (d), la densité des arbres, la couverture de la canopée (f) et la hauteur du nid (g). Les modèles candidats restants avaient un $\triangle \mathrm{AICc}>$ 3,16 et un $\boldsymbol{w}_{\boldsymbol{i}}<0,5$ pour l'espèce Eurillas latirostris et un $\Delta \mathrm{AICc}>4,73$ et un $\boldsymbol{w}_{\boldsymbol{i}}<0,04$ pour l'espèce Eurillas virens. 
Tableau 4: Influences des paramètres des sites de nidification sur le taux de prédation quotidien.

\begin{tabular}{|c|c|c|c|c|}
\hline \multirow[b]{2}{*}{ Paramètres } & \multicolumn{2}{|c|}{ Coefficient de régression } & \multirow{2}{*}{$\begin{array}{c}\text { Valeur } \\
\text { de } Z\end{array}$} & \multirow{2}{*}{$\begin{array}{c}\text { Valeur de } \\
\mathbf{P}(>|\mathbf{z}|)\end{array}$} \\
\hline & Estimation & $\begin{array}{c}\text { Erreur } \\
\text { standard }\end{array}$ & & \\
\hline \multicolumn{5}{|c|}{ Eurillas latirostris } \\
\hline Intercept & $-11,275$ & 4,539 & $-2,484$ & $0,013 *$ \\
\hline Surface terrière $\left(\mathbf{m}^{2} / \mathbf{h a}\right)$ & 0,134 & 0,56 & 2,39 & $0,016 *$ \\
\hline $\begin{array}{c}\text { Hauteur maximale de la } \\
\text { végétation au-dessus du nid }(\mathrm{cm})\end{array}$ & 0,044 & 0,029 & 1,527 & 0,127 \\
\hline \multicolumn{5}{|c|}{ Eurillas virens } \\
\hline Intercept & $-1,375 \mathrm{e}+01$ & $4,078 \mathrm{e}+03$ & $-0,003$ & 0,9973 \\
\hline Visibilité totale 3 & $-8,381 e+00$ & $2,055 \mathrm{e}+05$ & 0,000 & 1,0000 \\
\hline Visibilité totale 4 & $1,193 \mathrm{e}+01$ & $4,078 \mathrm{e}+03$ & 0,003 & 0,9977 \\
\hline Visibilité totale 5 & $1,489 \mathrm{e}+01$ & $4,078 \mathrm{e}+03$ & 0,004 & 0,9971 \\
\hline Visibilité totale 6 & $1,688 \mathrm{e}+01$ & $4,078 \mathrm{e}+03$ & 0,004 & 0,9967 \\
\hline Visibilité totale 7 & $1,279 \mathrm{e}+01$ & $4,078 \mathrm{e}+03$ & 0,003 & 0,9975 \\
\hline Visibilité totale 8 & $1,629 \mathrm{e}+01$ & $4,078 \mathrm{e}+03$ & 0,004 & 0,9968 \\
\hline $\begin{array}{c}\text { Hauteur maximale de la } \\
\text { végétation au-dessus du nid }(\mathrm{cm})\end{array}$ & $-4,827 \mathrm{e}-02$ & $1,983 \mathrm{e}-02$ & $-2,434$ & $0,0149 *$ \\
\hline
\end{tabular}
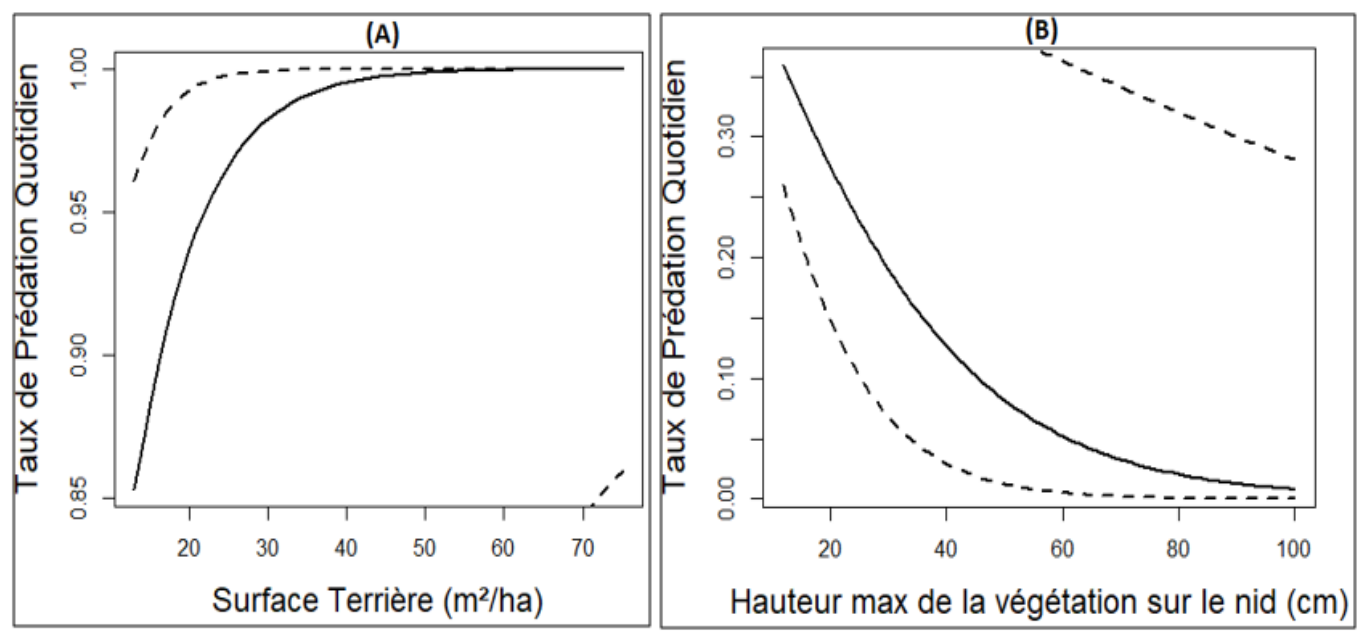

Figure 2: Influence des paramètres de la végétation sur le taux de prédation quotidien (TPQ) des nids des espèces Eurillas latirostris (A) et Eurillas virens (B). La ligne continue représente le TPQ estimé obtenu à partir du modèle logit-linéaire avec des co-variables significatives du meilleur modèle estimé. Les lignes pointillées représentent les intervalles de confiance supérieurs et inférieurs au seuil de 95\%. 


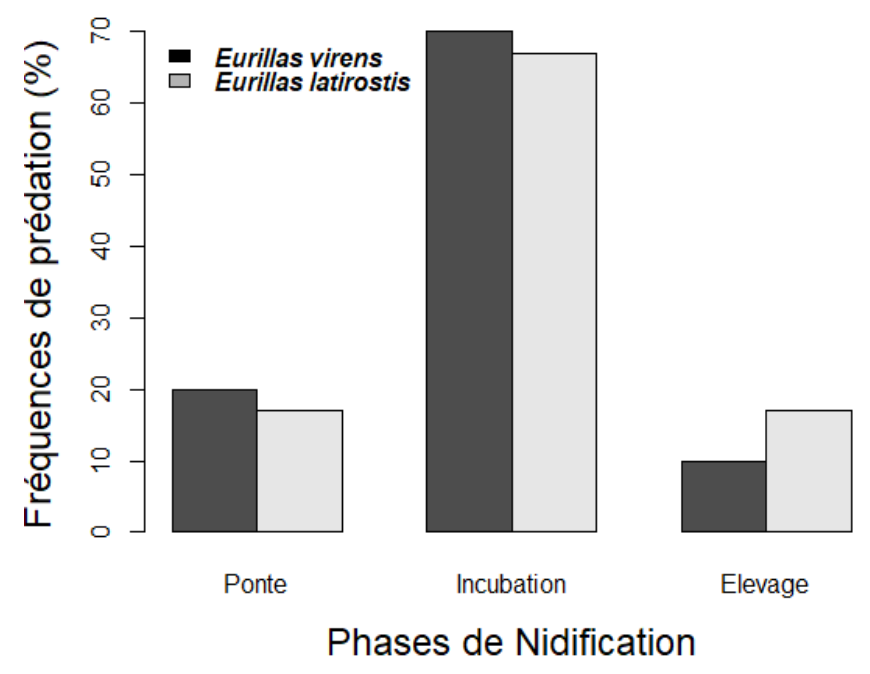

Figure 3: Taux de prédation suivant les phases de nidification.

\section{DISCUSSION}

\section{Taux de prédation des nids suivant les types d'habitat}

En général, la prédation est la cause principale de l'échec de nidification observé chez les espèces Eurillas latirostris et Eurillas virens avec plus de la moitié des nids attaqués. Ce taux de prédation est inférieur à celui observé dans des écosystèmes similaires des paysages tropicaux (Githiru et al., 2005; Ryder et al., 2008; Newmark et Stanley, 2011) et subtropicaux (Fu et al., 2016). La prédation des œufs est la plus courante, mais celle des oisillons est aussi observée dans une moindre mesure. Des résultats similaires ont été obtenus dans les zones tempérées (Debus, 2006; Tewksbury et al., 2006) et les régions tropicales (Githiru et al., 2005; Newmark et Stanley, 2011). Plusieurs raisons peuvent expliquer cette situation dont la diversité des prédateurs. En effet, les modèles de prédation dépendent de la réponse des différentes espèces de prédateurs (Bobo, 2007) à la composition du paysage, et des effets relatifs de ces prédateurs sur les espèces d'oiseaux.
Ceci à cause de la nature complexe de ces interactions, la relation universelle entre la fragmentation et la prédation qui est peu probable (Tewksbury et al., 1998). Par ailleurs, les types d'habitat n'ont pas d'influence significative sur les proportions des nids ayant subi la prédation ainsi que sur les taux de prédation des nids des deux espèces. Cependant, ce modèle de prédation contredit celui de Pangau-Adam et al. (2006) qui trouvait que le taux de prédation des nids s'accrôit avec la perturbation de l'écosystème. Ceci est dû à l'afflux des prédateurs des habitats voisins à cause des meilleures conditions créées par la dégradation de l'environnement des oiseaux qui favorisent ainsi une grande visibilité pour les prédateurs dans les habitats perturbés par rapport aux habitats intacts (Pangau-Adam et al., 2006; Tewksbury et al., 2006). Toutefois, la présente zone d'étude est différente des autres sites du fait que les habitats dominants sont des forêts (forêts primaires et forêts secondaires) en termes de superficies et de distributions, et sont peu perturbées, conduisant ainsi à un effet 
contraire (une forme d'exception à la règle). Ainsi, les prédateurs arrivent plutôt des zones dégradées, créant ainsi un surnombre dans les zones intactes ou bien les prédateurs de ces oiseaux restent plus confinés dans les zones très peu perturbées.

\section{Influences de la prédation par les variables de la végétation}

Généralement, la qualité de l'habitat semble affecter le succès de reproduction (Debus, 2006; Pangau-Adam et al., 2006) et les oiseaux sont, la plupart de temps, très scrupuleux dans le choix de leurs sites de nidification (Tonleu et al., 2018). Ceci explique pourquoi les perturbations liées aux activités anthropiques affectent la qualité des habitats car elles suppriment les éléments clés pour les individus nidificateurs (Debus, 2006). Comme dans les études de Fu et al. (2016) dans des écosystèmes subtropicaux, la présente étude a montré que certaines variables des sites de nidification influencent le TPQ des nids des deux espèces étudiées. Ainsi, la surface terrière et la hauteur maximale de la végétation au-dessus du nid sont les seules variables qui influencent significativement le TPQ des espèces Eurillas latirostris et Eurillas virens respectivement, bien que les actions des deux variables étaient différentes en termes d'intensité sur les différents TPQ. Cependant, l'action positive de la surface terrière sur le TPQ de l'espèce Eurillas latirostris, concentré dans l'intervalle 0,85 à 1 (Figure 2), a été constatée. Ceci démontre aussi indirectement que le taux de prédation est plus élevé dans les forêts naturelles car ces dernières possèdent les plus grandes valeurs moyennes des surfaces terrières (voir Tableau 2). Ceci implique aussi que les oiseaux choisissent toujours le meilleur site de nidification quel que soit le degré de perturbation (Tonleu et al., 2018). Ces résultats suggèrent dans une certaine mesure que les prédateurs des nids d'oiseaux du sousbois sont plus actifs dans les habitats relativement stables comme la forêt primaire et la forêt secondaire.

\section{Taux de prédation suivant les phases de la période de nidification}

Les modèles de prédation des nids des espèces Eurillas latirostris et Eurillas virens le long de la période de nidification ont été les mêmes. Ainsi, les proportions de prédation de ces deux espèces sont largement élevées durant la phase d'incubation et relativement basses pendant les phases de ponte et d'élevage des jeunes. Des résultats similaires ont été retrouvés chez certains oiseaux du sous-bois dans les écosystèmes similaires en Amérique du Sud (Ryder et al., 2008) ainsi que dans les forêts boréales en Europe (Cresswell, 1997). En revanche, certaines études ont tout de même montré que le TPQ augmentait plutôt d'une phase à une autre le long de la période de nidification (Ryder et al., 2008; Brawn et al., 2011; Fu et al., 2016; Jiang et al., 2017). Cette augmentation du taux de prédation pendant la phase d'incubation peut être expliquée par le relâchement de l'attention des adultes au début de la phase d'incubation (Tonleu et al., 2008). Cette situation suggère aussi que la perte des nids peut aussi être attribuée aux prédateurs qui utilisent les signaux visuels pour détecter leurs proies. En outre, les faibles proportions de taux de prédations enregistrées durant les phases de ponte et d'élevage des oisillons suggèrent que malgré que le nombre de visites sur le nid augmente pendant la période d'élevage, les oisillons restent complètement silencieux même pendant les moments de nutrition (Ryder et al., 2008). 


\section{Conclusion}

Le but principal de la présente étude était d'estimer le taux de prédation des nids des espèces Eurillas latirostris et Eurillas virens suivant les habitats naturelles et perturbés et les phases de nidification. Ainsi, il a été trouvé que plus de la moitié des nids des espèces Eurillas latirostris et Eurillas virens a subi la prédation bien que le taux de prédation soit inférieur à ceux obtenus dans les zones tempérées et arides. Aussi, certaines variables de la végétation (Surface terrière et couvert de la végétation au-dessus du nid) ont des influences significatives sur les TPQ. Toutefois, ces taux de prédation et les TPQ n'ont pas été affectés par les types d'habitats. En outre, les taux de prédation ont varié significativement d'une phase de nidification à une autre. Les variations des TPQ de ces espèces sont entraînées d'une part par la surface terrière et le couvert de la végétation au-dessus du nid pour l'espèce Eurillas latirostris, et d'autre part par la visibilité totale et le couvert de la végétation au-dessus du nid pour l'espèce Eurillas virens. Ainsi, la préservation des paysages naturels et la pratique des techniques de coupe de bois à impacts réduits (Sist et al., 2003; Putz et al., 2008) participeront à la conservation des espèces d'oiseaux du sous-bois.

\section{CONFLIT D'INTERETS}

Les auteurs déclarent qu'ils n'ont aucun conflit d'intérêts.

\section{CONTRIBUTIONS DES AUTEURS}

JT a participé à la conception de l'étude, aux travaux de terrain, aux analyses statistiques et à la rédaction du manuscrit. KSB et MM ont participé à la conception de l'étude et à la rédaction du manuscrit. DWD, WGTL, ACT, UTN et LKM ont participé aux travaux sur le terrain CLTT a participé aux travaux sur le terrain et à la rédaction du manuscrit. RSHA a participé à l'analyse des données et à la rédaction du manuscrit. Tous les auteurs ont lu et approuvé le manuscrit final.

\section{REMERCIEMENTS}

Les auteurs remercient tous les habitants des trois villages (Mgbegati, Abat et Basu) autour de notre zone d'étude pour leurs contributions. Merci enfin à M. Hamman Missa, Dr Kougoum Pieben Ghislain, Dr Ndjeumi Chrisdel Chancelice et Dr Bayoi James et à quelques critiques anonymes pour leurs commentaires sur les versions préliminaires de ce manuscrit.

\section{REFERENCES}

Agresti A. 2002. Categorical Data Analysis (2nd edn). Wiley: New York; 91-101.

Bates D, Mächler M, Bolker B, Walker S. 2014. Fitting linear mixed-effects models using lme4. ArXiv Prepr. ArXiv14065823.

Bellamy PE, Burgess MD, Mallord JW, Cristinacce A, Orsman CJ, Davis T, Grice PV, Charman EC. 2017. Nest predation and the influence of habitat structure on nest predation of Wood Warbler Phylloscopus sibilatrix, a ground-nesting forest passerine. Journal of Ornithology, 2: 1-14. DOI: https://doi.org/10.1007/s10336-0171527-7

Bobo KS, Waltert M, Sainge NM, Njokagbor J, Fermon H, Mühlenberg M. 2006. From forest to farmland: species richness patterns of trees and understorey plants along a gradient of forest conversion in Southwestern Cameroon. Biodiversity and Conservation, 15(13): 4097-4117. DOI: https://doi.org/10.1007/s10531005-3368-6. 
Bobo KS, 2007. From forest to farmland: Effects of land use on understorey birds of Afrotropical rainforests. Thèse de Doctorat/PhD, Université de GeorgAugust- Göttingen.

Bobo KS, Waltert M, 2011. The importance of agricultural areas for bird conservation in the Korup region, southwestern Cameroon. International Journal of Biological and Chemical Sciences, 5(2): 419-432. DOI: http://dx.doi.org/10.4314/ijbcs.v5i2.7206 3

Borrow N, Demey R. 2008. Guide des oiseaux de l'Afrique de l'Ouest. Delachaux et Niestlé.

Brawn JD, Angehr G, Davros N, Robinson WD, Styrsky JN, Tarwater CE. 2011. Sources of variation in the nesting success of understory tropical birds. Journal of Avian Biology, 42(1): 61-68. DOI: $\quad$ https://doi.org/10.1111/j.1600048X.2010.04897.X

Buler JJ, Hamilton RB. 2000. Predation of natural and artificial nests in a southern pine forest. The Auk, 117: 739-747. DOI: https://doi.org/10.1642/0004-8038 (2000)117[0739: PONAAN] 2.0.CO; 2

Burnham KP, Anderson DR. 2003. Model Selection and Multimodel Inference: a Practical Information-theoretic Approach. Springer Science \& Business Media.

Clark DA, Clark DB. 1992. Life history diversity of canopy and emergent trees in a neotropical rain forest. Ecological Monographs, 62(3): 315-344. DOI: https://doi.org/10.2307/2937114

Conover WJ. 1972. A Kolmogorov goodnessof-fit test for discontinuous distributions. Journal of the American Statistical Association, 67(339): 591-596. DOI: 10.1080/01621459.1972.10481254
Cresswell W. 1997. Nest predation: the relative effects of nest characteristics, clutch size and parental behaviour. Animal Behaviour, 53(1): 93-103. DOI: https://doi.org/10.1006/anbe.1996.0281

Debus SJS. 2006. Breeding-habitat and nestsite characteristics of Scarlet Robins and Eastern Yellow Robins near Armidale, New South Wales. Pac. Conservation Biology, 12(4): 261-271. DOI: https://doi.org/10.1071/PC060261

Dion N, Hobson KA, Larivière S. 2000. Interactive effects of vegetation and predators on the success of natural and simulated nests of grassland songbirds. The Condor, 102(3): 629-634. DOI: https://doi.org/10.1650/0010-5422 (2000)102[0629: IEOVAP] 2.0.CO; 2

Ekanayake KB, Whisson DA, Tan LX, Weston MA. 2015. Intense predation of non-colonial, ground-nesting bird eggs by corvid and mammalian predators. Wildlife Research, 42(6): 518-528. DOI: https://doi.org/10.1071/WR15080

Farnsworth GL, Simons TR. 1999. Factors affecting nesting success of wood thrushes in Great Smoky Mountains National Park. The Auk, 116(4): 10751082. DOI: $10.2307 / 4089686$

Fishpool LD, Evans MI, others. 2001. Important Bird Areas in Africa and Associated Islands: Priority Sites for Conservation. BirdLife International: Cambridge, UK.

Fu Y, Chen B, Dowell SD, Zhang Z. 2016. Nest predators, nest-site selection and nest success of the Emei Shan Liocichla (Liocichla omeiensis), a vulnerable babbler endemic to southwestern China. Avian Research, 7(1): 18. DOI: 10.1186 /s40657-016-0054-1

Githiru M, Lens L, Cresswell W. 2005. Nest predation in a fragmented Afrotropical 
forest: evidence from natural and artificial nests. Biological Conservation, 123(2): $\quad 189-196 . \quad$ DOI: https://doi.org/10.1016/j.biocon.2004.11. 006

Hollander M, Wolfe DA, Chicken E. 2013. Nonparametric Statistical Methods. John Wiley \& Sons.

Jiang A, Jiang D, Zhou F, Goodale E. 2017. Nest-site selection and breeding ecology of Streaked Wren-Babbler (Napothera brevicaudata) in a tropical limestone forest of southern China. Avian Research, 8(1). DOI: 10.1186 /s40657017-0086-1

Kougoum PGN, Tamungang SA, Teguia A. 2017. Breeding biology of African grey parrot (Psittacus erithacus) in Kom National Park (South-Cameroon) and implications to the species conservation. International Journal of Biological and Chemical Sciences, 11(5): 1948-1966. DOI: https://dx.doi.org/10.4314/ijbcs.v11i5.2

Li P, Martin TE. 1991. Nest-site selection and nesting success of cavity-nesting birds in high elevation forest drainages. The Auk, 108(2): 405-418.

Martin TE. 1993. Nest predation and nest sites. BioScience, 43(8): 523.

Martinez WE, Piaskowski VD, Teul M. 2004. Reproductive biology of the Graycrowned Yellowthroat (Geothlypis poliocephala palpebralis) in central Belize. Ornitologia Neotropical, 15: 155-162.

Mayfield HF. 1975. Suggestions for calculating nest success. The Wilson Bulletin, 87(4): 456-466.

Mazerolle MJ. 2016. AICcmodavg: Model selection and multimodel inference based on (Q) AIC (c)[Software].
Mezquida ET. 2004. Nest site selection and nesting success of five species of passerines in a South American open Prosopis woodland. Journal of Ornithology, 145(1): 16-22. DOI: https://doi.org/10.1007/s10336-0030002-9

Mezquida ET, Marone L. 2001. Factors affecting nesting success of a bird assembly in the central Monte Desert, Argentina. Journal of Avian Biology, 32(4): 287-296. DOI: https://doi.org/10.1111/j.0908-

8857.2001.320401.x

Newmark WD, Stanley TR. 2011. Habitat fragmentation reduces nest survival in an Afrotropical bird community in a biodiversity hotspot. Proceedings of the National Academy of Sciences, 108(28): 11488-11493. DOI: https://doi.org/10.1073/pnas.1104955108

Newton A. 2007. Forest Ecology and Conservation: A Handbook of Techniques (Techniques in Ecology \& Conservation), 1st ed. Oxford University Press.

Pangau-Adam MZ, Waltert M, Mühlenberg M. 2006. Nest predation risk on ground and shrub nests in forest margin areas of Sulawesi, Indonesia. Biodiversity and Conservation, 15(13): 4143-4158. DOI: https://doi.org/10.1007/s10531-0053370-z

Putz FE, Sist P, Fredericksen T, Dykstra D. 2008. Reduced-impact logging: challenges and opportunities. Forest Ecology and Management, 256(7): 1427-1433.

DOI: https://doi.org/10.1016/j.foreco.2008.03. 036

Remeš V. 2005. Nest concealment and parental behaviour interact in affecting nest survival in the blackcap (Sylvia 
atricapilla): an experimental evaluation of the parental compensation hypothesis. Behavioral Ecology and Sociobiology, 58(3): $\quad 326-332 . \quad$ DOI: https://doi.org/10.1007/s00265-0050910-1

Robinson WD, Robinson TR, Robinson SK, Brawn JD. 2000. Nesting success of understory forest birds in central Panama. Journal of Avian Biology, 31(2): $\quad$ 151-164. DOI: https://doi.org/10.1034/j.1600048X.2000.310207.x

Rodewald AD, Yahner RH. 2001. Avian nesting success in forested landscapes: influence of landscape composition, stand and nest-patch microhabitat, and biotic interactions. The Auk, 118(4): 1018-1028. DOI: https://doi.org/10.1642/0004-8038 (2001)118[1018: ANSIFL] 2.0.CO; 2

Rodewald PG, Dejaifve P-A, Green AA. 1994. The birds of Korup National Park and Korup Project Area, Southwest Province, Cameroon. Bird Conservation International, 4(1): 1-68. DOI: https://doi.org/10.1017/S0959270900002 $63 \mathrm{X}$

Ryder TB, Durães R, Tori WP, Hidalgo JR, Loiselle BA, Blake JG. 2008. Nest survival for two species of manakins (Pipridae) in lowland Ecuador. Journal of Avian Biology, 39(3): 355-358. DOI: https://doi.org/10.1111/j.09088857.2008.04290.x

Scherrer B. 1984. Biostatistique. Gaëtan Morin.

Shaffer TL. 2004. A unified approach to analyzing nest success. The Auk, 121(2): 526-540.

DOI:

https://doi.org/10.1642/0004-8038

(2004)121[0526: AUATAN] 2.0.CO; 2
Sist P, Sheil D, Kartawinata K, Priyadi H. 2003. Reduced-impact logging in Indonesian Borneo: some results confirming the need for new silvicultural prescriptions. Forest Ecology and Management, 179(1): 415-427. DOI: https://doi.org/10.1016/S0378-1127 (02)00533-9

Symonds MR, Moussalli A. 2011. A brief guide to model selection, multimodel inference and model averaging in behavioural ecology using Akaike's information criterion. Behavioral Ecology and Sociobiology, 65(1): 13-21. DOI: https://doi.org/10.1007/s00265010-1037-6

Tewksbury JJ, Garner L, Garner S, Lloyd JD, Saab V, Martin TE. 2006. Tests of landscape influence: nest predation and brood parasitism in fragmented ecosystems. Ecology, 87(3): 759-768. DOI: https://doi.org/10.1890/04-1790

Tewksbury JJ, Hejl SJ, Martin TE. 1998. Breeding productivity does not decline with increasing fragmentation in a western landscape. Ecology, 79(8): 2890-2903.

DOI:

https://doi.org/10.1890/0012-9658

(1998)079[2890: BPDNDW] 2.0.CO; 2

Thomas D. 1995. Botanical Survey of the Rumpi Hills and Nta Ali. Report to Korup Project.

Tonleu J, Bobo KS, Djoumessi WD, Mbida M. 2018. Understorey birds' nests predation in afrotropical forest ecosystem: A case study of Korup area, Cameroon. Journal of Ecology and The Natural Environment, 10(8): 192-204. DOI:

https://doi.org/10.5897/JENE2018.0707

Venables WN, Ripley BD. 2002. Modern Applied Statistics with $S$ | W.N. 
Venables | Springer. Springer, Oxford OX1 3TG Australia England bill.

Vergara PM, Simonetti JA. 2004. Does nestsite cover reduce nest predation for rhinocryptids? Journal of Field Ornithology, 75(2): 188-191. DOI: https://doi.org/10.1648/0273-857075.2.188.

Vergara PM, Simonetti JA. 2003. Forest fragmentation and rhinocryptid nest predation in central Chile. Acta Oecologica, 24(5): 285-288. DOI: https://doi.org/10.1016/j.actao.2003.09.0 06

Weidinger K. 2000. The breeding performance of blackcap Sylvia atricapilla in two types of forest habitat. Ardea, 88(2): 225-233.

Wray TII, Whitmore RC. 1979. Effects of vegetation on nesting success of Vesper Sparrows. The Auk, 96(4): 802-805.

Yandell BS. 1997. Practical Data Analysis for Designed Experiments, Chapman \& Hall Texts in Statistical Science Series. Springer US.

Yates F. 1934. Contingency tables involving small numbers and the $\chi 2$ test. Supplement of the Journal of the Royal Statistical Society, 1(2): 217-235. DOI: 10.2307/2983604 